\title{
Artropodofauna cadavérica asociada a cerdo doméstico (Sus scrofa) en un ambiente ribereño en Chubut, Argentina
}

\author{
Fauna carrion associated to domestic pig (Sus scrofa) in a coastal environment in Chubut, Argentina
}

\author{
ANA PAULA ARMANI ${ }^{1}$, SILVIA DAHINTEN ${ }^{2}$ y NESTOR CENTENO ${ }^{3}$
}

\begin{abstract}
Resumen: Este es el primer estudio de descomposición cadavérica realizado en un ambiente ribereño en la región NO de la provincia de Chubut Argentina, cuyo objetivo fue determinar la composición específica, la abundancia y la diversidad de la artropodofauna cadavérica asociada a un modelo experimental porcino. Se realizaron muestreos estacionales durante tres años consecutivos (2010 - 2012) utilizando en cada muestreo tres cerdos, colocados dentro de jaulas de madera cubiertas de alambre tejido. Diariamente se colectaron los insectos en el cuerpo, debajo y alrededor de este. Se identificaron y caracterizaron las cinco etapas del proceso de descomposición de los cadáveres de cerdo experimentales. Se recolectaron 6.325 individuos adultos e inmaduros pertenecientes a 63 especies de 18 familias distribuidas entre los órdenes Diptera, Coleoptera e Hymenoptera. Se determinó la estacionalidad de Compsomyiops fulvicrura (Diptera: Calliphoridae) durante la época cálida y la dominancia de Calliphora vicina (Diptera: Calliphoridae) en la época fría. Los resultados obtenidos aportan información relevante sobre la fauna de insectos saprófagos en el ambiente ribereño al río Chubut, que puede ser utilizada como herramienta en la estimación de la data de muerte en casos forenses humanos en la región estudiada.
\end{abstract}

Palabras clave: Entomología forense, fauna cadavérica, insectos sarcosaprófagos, Patagonia.

\begin{abstract}
This is the first study of cadaveric decomposition taking place in a riverside environment of the NW of Chubut province, Argentina. The specific composition, abundance, diversity of the carrion fauna associated with a porcine experimental model was determined. Seasonal samplings were performed for three consecutive years (2010-2012) using three domestic pigs for each sample, placed in wooden cages covered with wire mesh. Daily, insects were collected in the body, under and around it. There were identified and characterized the five stages of bodies' decomposition of experimental pigs. Were collected 6,325 individuals adult and immature of 63 species belonging to 18 families distributed among the Diptera, Coleoptera and Hymenoptera orders. During the warm season, was determined the seasonality of Compsomyiops fulvicrura (Diptera: Calliphoridae) and, the dominance of Calliphora vicina (Diptera: Calliphoridae) in the cold season. Relevant information on the saprophagous insects in the riverside environment Chubut, which is helpful in estimating the time of death in forensic cases in the study region.
\end{abstract}

Key words: Forensic entomology, cadaveric fauna, saprophagous insects, Patagonia.

\section{Introducción}

La entomología forense tiene como objeto de estudio a la fauna de artrópodos que coloniza un cadáver. Su práctica profesional vinculada al aspecto médico-legal implica la realización de pericias que aportan información en la investigación de causas judiciales por homicidio, muertes dudosas u otras figuras jurídicas. La pericia entomológica contribuye a establecer el intervalo postmortem (PMI) y las condiciones del entorno en las que se produjo el deceso (Oliva 1997). El obtener información oportuna y fiable es vital para incriminar o exonerar a las partes involucradas, de ahí deriva la importancia de la interpretación de la entomofauna asociada al cadáver, que puede ser ignorada por las autoridades quienes no la consideran como parte de la evidencia (Byrd y Castner 2001).

Dado que los artrópodos se encuentran ampliamente distribuidos y poco tiempo después de la muerte llegan al cuerpo, son útiles para determinar las circunstancias, lugar y tiempo ocurrido desde el fallecimiento hasta el hallazgo del cadáver (Benecke 2001; Marchenko 2001). Sin embargo, para obtener una cifra acertada del intervalo postmortem, se deben considerar las condiciones ambientales del área y ciertos principios básicos de la colonización de un cuerpo por los insectos, la cual sigue un patrón predecible de sucesión de especies (Dadour et al. 2001). Además, las condiciones meteorológicas, principalmente temperatura y humedad rela- tiva, influyen sobre la presencia y ausencia, abundancia y/o actividad de las especies de insectos que colonizan un cadáver en determinada región (Castillo-Miralbés 2002; Tabor et al. 2005).

En Argentina, son numerosos los estudios realizados sobre la comunidad sarcosaprófaga, principalmente en localidades del centro y norte del país como Córdoba (Battán Horenstein et al. 2005; Battán Horenstein et al. 2007; Battán Horenstein et al. 2010; Battán Horenstein y Linhares 2011; Battán Horenstein y Salvo 2012; Battán Horenstein et al. 2012), Buenos Aires (Oliva 1997; Centeno et al. 2002; Mariani et al. 2014; Zanetti et al. 2015), Mendoza, (Aballay et al. 2012), San Juan (Aballay et al. 2008, Aballay et al. 2012), Catamarca (Aballay et al. 2011) y Salta (Ayón et al. 2004; Ayón 2013). Sin embargo, existen escasos trabajos realizados sobre descomposición y artropodofauna cadavérica en la Patagonia Argentina (Armani et al. 2015). El objetivo de este trabajo fue determinar la composición específica, abundancia y diversidad de la artropodofauna en un cerdo doméstico del ambiente ribereño, característico de la región noreste de la provincia del Chubut, Argentina.

\section{Materiales y métodos}

Sitio de estudio. El trabajo se realizó en la ciudad de Trelew, provincia de Chubut, Argentina ( $43^{\circ} 16^{\prime} 15,6^{\prime \prime} \mathrm{S}$ y $65^{\circ} 15^{\prime} 0,9^{\prime \prime} \mathrm{O}$ a $11 \mathrm{msnm}$ ) en una parcela experimental situada en el Valle

\footnotetext{
${ }^{1}$ Licenciada en Ciencias Biológicas. Instituto de Diversidad y Evolución Austral (IDEAus) CONICET. Boulevard Brown No 2915 Puerto Madryn, Chubut (Argentina), ana_armani@hotmail.com, autor para correspondencia. ${ }^{2}$ Dra. Silvia Lucrecia Dahinten. Instituto de Diversidad y Evolución Austral (IDEAus) CONICET, dahinten@cenpat-conicet.gob.ar. ${ }^{3}$ Dr. Néstor Daniel Centeno. Universidad Nacional de Quilmes, Buenos Aires, Argentina,ncenteno@unq.edu.ar.
} 
Inferior del río Chubut, a $50 \mathrm{~m}$ del mencionado río. El clima es templado-frío y ventoso, no se observa un régimen de lluvias definido. La temperatura mínima es de $-12{ }^{\circ} \mathrm{C}$ y máxima de $38^{\circ} \mathrm{C}$ con presencia de heladas (Owen et al. 2005).

Fase de campo. Los muestreos se realizaron durante tres años consecutivos quedando representadas las cuatro estaciones: primavera (octubre - noviembre 2010), verano (febrero-abril 2011), invierno (julio - noviembre 2011) y otoño (abril-octubre 2012). No se realizaron los muestreos estacionales en un mismo año debido a que se solaparían los experimentos, ya que el proceso de descomposición demora en general, más de un mes. En cada muestreo se utilizaron como modelo experimental tres cerdos domésticos (entre $12-16 \mathrm{~kg}$ ) sacrificados por el método de faena comercial (Centeno et al. 2002). En el sitio de estudio cada cerdo fue colocado dentro de una jaula de madera, cubierta de alambre tejido de un tamaño de $1 \frac{1 / 4}{4}$ pulgada y madera para evitar la actividad de vertebrados carroñeros, pero permitir que los insectos puedan acceder al cuerpo. Alrededor de cada jaula se colocaron seis trampas de caída o "pitfall" que contenían etilenglicol al $30 \%$ como líquido fijador y conservador. Estas trampas se utilizaron para colectar los insectos y demás artrópodos que arriban al cuerpo y migran del mismo. Así mismo, se colocaron seis trampas de caída en un área control alejadas $50 \mathrm{~m}$ de los cerdos experimentales, para obtener una muestra control de insectos del área de estudio. Sobre los cerdos experimentales se recolectaron los insectos con pinzas y red entomológica. Para registrar las características de las diferentes etapas del proceso de descomposición, se tomaron diariamente notas y fotografías utilizando una cámara digital KODAK DX7590. Los datos de temperatura máxima, mínima y humedad relativa ambiental se obtuvieron de un termómetro higrómetro digital HERTER 5757. Durante los primeros días, y hasta que el estado de descomposición de los cuerpos lo permitió, se registró la temperatura corporal interna de cada cerdo con un termómetro digital de punción Marca LUFT. La frecuencia de muestreo fue diaria hasta que los modelos experimentales alcanzaron el estado de restos. Posteriormente se realizó cada $2 / 3$ días hasta que no se observó actividad de insectos en los cerdos.

Se identificaron las cinco etapas del proceso de descomposición descritas por Payne (1965) y, Anderson y VanLaerhoven (1996): fresco (F), hinchado (H), descomposición activa (DAC), descomposición avanzada (DAZ) $\mathrm{y}$ restos $(\mathrm{R})$.

Fase de laboratorio. El material recolectado se trasladó al laboratorio y se conservó en alcohol etílico al $70 \%$. Para su identificación se utilizaron claves taxonómicas especializadas. Dicho material forma parte de una colección entomológica de referencia que fue depositada en el Centro Nacional Patagónico (CENPAT - CONICET) de la ciudad de Puerto Madryn, Chubut (Argentina).

Análisis estadísticos. Para analizar la diversidad de insectos recolectados durante los muestreos estacionales se utilizaron los índices de diversidad de Shannon $(\mathrm{H})$ y de riqueza de Margalef mediante el programa estadístico PAST. Se realizó un análisis entre las estaciones del año y los índices de diversidad de Shannon y de riqueza de Margalef utilizando el programa estadístico antes mencionado.

\section{Resultados}

Estados de descomposición. Se observaron las cinco etapas del proceso de descomposición: fresco $(\mathrm{F})$, hinchado $(\mathrm{H})$, descomposición activa (DAC), descomposición avanzada (DAZ) y restos (R). En general, la etapa fresca fue la de menor duración en días. En el invierno y otoño todas las etapas tuvieron una mayor duración. Para el otoño la etapa de restos fue más larga (129 días) que en las demás estaciones del año (Fig. 1).

La duración total del proceso de descomposición (Fig. 1) fue mayor para el otoño (181 días), seguida por el invierno (100 días) y el verano (64 días). En tanto en la primavera, el proceso de descomposición fue más corto (43 días) que para el resto de las estaciones.

Elenco específico. Se recolectaron un total de 6.325 individuos (Tabla 1) adultos e inmaduros, pertenecientes a 63 especies de 18 familias distribuidas entre los órdenes Diptera, Coleoptera e Hymenoptera, siendo los más importantes y numerosos desde el punto de vista de la entomología forense.

Durante el verano se observó la mayor abundancia total de individuos $(\mathrm{N}=2.166)$ seguida por el otoño $(\mathrm{N}=2.132)$. En tanto que la primavera fue la estación con menor abundancia $(\mathrm{N}=850)$.

La menor diversidad de especies de insectos cadavéricos se observa durante otoño mientras que en cuanto a la riqueza específica, se observó el mayor valor del índice de Margalef para el verano (Tabla 2).

Durante el invierno y la primavera se observó la mayor similitud entre los índices de Shannon y Margalef, mientras que el verano difiere considerablemente de las demás estaciones (Fig. 2).

\section{Discusión}

Las etapas del proceso de descomposición halladas en el presente trabajo muestran similares características a estudios realizados en Latinoamérica (Wolff et al. 2001; Martínez et al. 2007; Salazar Ortega 2008; Segura et al. 2009, 2011). La duración total del proceso de descomposición en Chubut para primavera, verano y otoño fue mayor respecto a las provincias de Salta (Ayón et al. 2004), Córdoba (Battán Horenstein et al. 2010) y Buenos Aires (Centeno et al. 2002). En cambio

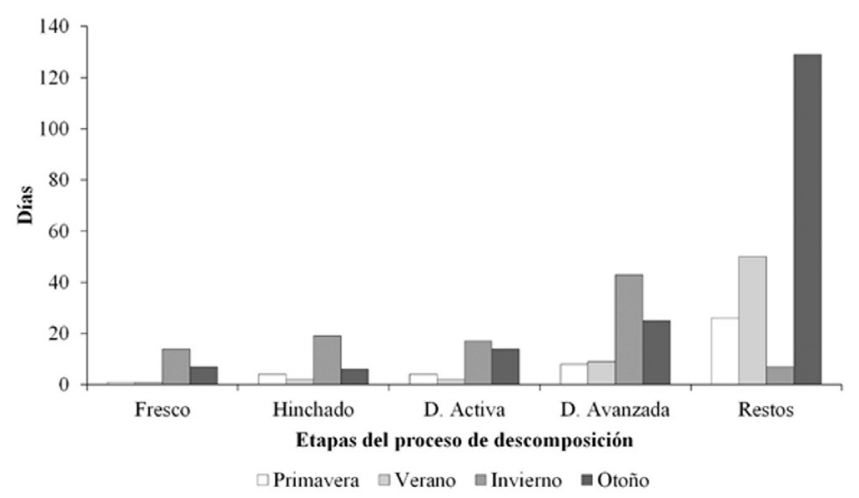

Figura 1. Duración (días) de las etapas del proceso de descomposición durante las cuatro estaciones del año. 
Tabla 1. Composición específica y abundancia total de las especies colectadas durante las cuatro estaciones. Referencias Pr: primavera, V: verano, I: invierno, O: otoño, A: adulto, LII: larva II, LIII: larva III, P: pupario.

\begin{tabular}{|c|c|c|c|c|c|c|}
\hline Orden & Familia & Especie / Morfoespecie & Pr & $\mathbf{V}$ & $\mathbf{O}$ & I \\
\hline & Calliphoridae & Calliphora vicina Robineau-Desvoidy (A) & 18 & 3 & 76 & 175 \\
\hline & & C. vicina $(\mathrm{LIII})$ & 0 & 0 & 0 & 255 \\
\hline & & C. vicina $(\mathrm{P})$ & 0 & 0 & 0 & 1 \\
\hline & & Lucilia sericata Meigen (A) & 38 & 28 & 27 & 4 \\
\hline & & L. sericata (LII) & 0 & 43 & 0 & 0 \\
\hline & & L. sericata (LIII) & 112 & 182 & 845 & 9 \\
\hline & & L. sericata $(\mathrm{P})$ & 0 & 0 & 5 & 0 \\
\hline & & Chrysomya albiceps Wiedemann (A) & 0 & 35 & 4 & 0 \\
\hline & & C. albiceps (LIII) & 0 & 12 & 0 & 0 \\
\hline & & C. albiceps $(\mathrm{P})$ & 0 & 1 & 2 & 0 \\
\hline & & Compsomyiops fulvicrura Robineau-Desvoidy (A) & 189 & 835 & 5 & 7 \\
\hline & & Compsomyiops sp. (LII) & 0 & 137 & 0 & 0 \\
\hline & & Compsomyiops sp.(LIII) & 254 & 306 & 0 & 1 \\
\hline & & Compsomyiops sp. (P) & 0 & 10 & 2 & 2 \\
\hline & & Sarconesia chlorogaster Wiedemann (A) & 16 & 1 & 1 & 0 \\
\hline & & S. chlorogaster $(\mathrm{P})$ & 1 & 1 & 0 & 0 \\
\hline & & Chlorobrachycoma versicolor Bigot (A) & 0 & 1 & 0 & 0 \\
\hline & Sarcophagidae & Oxysarcodexia terminalis Wiedemann (A) & 0 & 1 & 0 & 0 \\
\hline & & Oxysarcodexia varia Walker (A) & 1 & 2 & 0 & 0 \\
\hline & & Oxysarcodexia paulistanensis Mattos (A) & 0 & 0 & 3 & 0 \\
\hline & & Ravinia aureopyga Hall (A) & 6 & 6 & 1 & 0 \\
\hline \multirow[t]{30}{*}{ Diptera } & & Sarcophaga (Bercaea) africa Wiedemann (A) & 0 & 4 & 0 & 0 \\
\hline & & Sarcophaga (Liopygia) argyrostoma Robineau-Desvoidy (A) & 0 & 1 & 0 & 0 \\
\hline & & Sarcophaga (Liopygia) crassipalpis Macquart (A) & 0 & 0 & 2 & 0 \\
\hline & & Microcerella acrydiorum Weyenbergh (A) & 1 & 1 & 1 & 0 \\
\hline & & Microcerella chilena Dodge (A) & 1 & 0 & 0 & 0 \\
\hline & & Microcerella sp. Macquart (A) & 0 & 0 & 1 & 0 \\
\hline & & Microcerella sp. 2 (A) & 0 & 0 & 2 & 0 \\
\hline & & Microcerella sp. 4 (A) & 0 & 0 & 1 & 0 \\
\hline & & Sarcophagidae (LIII) & 0 & 1 & 0 & 0 \\
\hline & Muscidae & Musca domestica Linnaeus (A) & 3 & 77 & 4 & 0 \\
\hline & & Muscina stabulans Fallen (A) & 0 & 2 & 1 & 0 \\
\hline & & Ophyra aenescens Wiedemann (A) & 0 & 1 & 0 & 2 \\
\hline & & Arthurella choelensis Patitucci \& Mariluis (A) & 0 & 23 & 3 & 0 \\
\hline & & sp. 1 (A) & 0 & 0 & 0 & 1 \\
\hline & & sp. 2 (A) & 0 & 0 & 0 & 6 \\
\hline & Phoridae & sp. 1 (A) & 1 & 15 & 123 & 102 \\
\hline & Piophilidae & sp. 1 (A) & 8 & 3 & 0 & 1 \\
\hline & Scatopsidae & sp. 1 (A) & 1 & 0 & 790 & 250 \\
\hline & Fanniidae & Fannia canicularis Linnaeus (A) & 0 & 8 & 1 & 1 \\
\hline & & sp. $1(\mathrm{~A})$ & 3 & 23 & 19 & 19 \\
\hline & & sp. 2 (A) & 81 & 3 & 27 & 2 \\
\hline & & sp.3 (A) & 0 & 0 & 27 & 11 \\
\hline & & sp. 5 (A) & 0 & 73 & 0 & 0 \\
\hline & & sp. $8(A)$ & 0 & 5 & 0 & 0 \\
\hline & Stratiomydae & sp. 1 (A) & 0 & 0 & 2 & 0 \\
\hline & Syrphidae & sp.1 (A) & 0 & 0 & 1 & 0 \\
\hline & Rhinophoridae & Stevenia deceptoria Loew (A) & 0 & 131 & 0 & 0 \\
\hline & Dermestidae & Dermestes maculatus DeGeer (A) & 3 & 49 & 23 & 50 \\
\hline & & Dermestes sp. 2 (A) & 0 & 0 & 0 & 2 \\
\hline & & Dermestes sp. (L) & 0 & 36 & 0 & 3 \\
\hline
\end{tabular}




\begin{tabular}{|c|c|c|c|c|c|c|}
\hline Orden & Familia & Especie / Morfoespecie & Pr & $\mathbf{V}$ & $\mathbf{O}$ & $\mathbf{I}$ \\
\hline & Cleridae & Necrobia rufipes DeGeer (A) & 2 & 26 & 5 & 4 \\
\hline & Carabidae & Cnemalobus sp. Guerin-Meneville (A) & 2 & 0 & 0 & 0 \\
\hline & & sp. $1(\mathrm{~A})$ & 1 & 0 & 4 & 10 \\
\hline \multirow[t]{17}{*}{ Coleoptera } & & sp. 2 (A) & 1 & 0 & 0 & 5 \\
\hline & & sp. 3 (A) & 1 & 0 & 0 & 0 \\
\hline & Tenebrionidae & Scotobius pilularius Germar (A) & 37 & 11 & 49 & 40 \\
\hline & & Sp. 6 (A) & 0 & 1 & 0 & 0 \\
\hline & Histeridae & Euspilotus lacordairei Marseul (A) & 1 & 3 & 0 & 5 \\
\hline & & Euspilotus modestus Erichson (A) & 1 & 2 & 0 & 0 \\
\hline & & Euspilotus ornatus Blanchard (A) & 5 & 0 & 0 & 2 \\
\hline & Staphylinidae & Creophilus maxillosus Linnaeus (A) & 1 & 4 & 1 & 24 \\
\hline & & Philonthus rubromaculatus Bernhauer (A) & 0 & 3 & 0 & 0 \\
\hline & & Philonthus rectangularis Leech (A) & 3 & 1 & 0 & 0 \\
\hline & & Philonthus bonaerensis Bernhauer (A) & 0 & 2 & 0 & 0 \\
\hline & & Philonthus sp. Stephens (A) & 0 & 4 & 4 & 8 \\
\hline & & sp. 1 (A) & 0 & 1 & 3 & 5 \\
\hline & & sp. 2 (A) & 0 & 0 & 0 & 13 \\
\hline & & sp. 3 (A) & 0 & 3 & 4 & 0 \\
\hline & & sp. $4(\mathrm{~A})$ & 0 & 1 & 1 & 0 \\
\hline & Antichidae & Antichidae Latreille (A) & 0 & 0 & 39 & 108 \\
\hline \multirow[t]{5}{*}{ Hymenoptera } & Formicidae & Camponotus Mayr & 1 & 0 & 0 & 0 \\
\hline & & Dorymyrmex wolfhügeli Forel & 35 & 0 & 0 & 0 \\
\hline & & Dorymyrmex ensifer Forel & 9 & 0 & 0 & 0 \\
\hline & & Dorymyrmex Mayr & 0 & 37 & 0 & 15 \\
\hline & & Solenopsis Westwood & 13 & 7 & 23 & 34 \\
\hline Total & & & 850 & 2166 & 2132 & 1177 \\
\hline
\end{tabular}

para el invierno la duración de la descomposición fue menor en Chubut respecto a Salta (Ayón et al. 2004) pero de mayor duración que las de Buenos Aíres (Centeno et al. 2002) y Córdoba (Battán Horenstein et al. 2010).

El ensamble de Calliphoridae recolectado del ambiente ribereño, incluye a todas las especies citadas para la provincia de Chubut por Mariluis y Mulieri (2003).

Chrysomya albiceps Wiedemann es una especie invasora cuyo límite sur de distribución lo constituía la localidad de Viedma $\left(40^{\circ} 48^{\prime} \mathrm{S} 63^{\circ} 00^{\prime} \mathrm{O}\right)$ provincia de Río Negro (Centeno 1998), aunque Oliva (1977), menciona pupas de Chrysomyia Robineau-Desvoidy, asociadas a cadáveres de Otaria flavescens (Shaw, 1800) en Punta Ninfas (4256'14'’S 64²0'05'O) provincia de Chubut (Mariluis y Mulieri 2003). Asimismo, dicha especie fue citada dentro del ensamble de Calliphoridae asociada a cerdos domésticos en trabajos de descomposición en un ambiente de estepa en Chubut, Argentina (Armani et al. 2015). En Chubut y Buenos Aires, C. albiceps está presente en otoño y verano (Centeno et al. 2002) aunque no domina en ninguna estación. Mientras que en Salta (Ayón et al. 2004) y Córdoba (Battán Horenstein et al. 2010) es la especie más abundante en ambas estaciones.

Calliphora vicina Robineau-Desvoidy está presente durante todo el año en el ambiente ribereño, siendo dominante durante el invierno. Esta especie se encuentra presente en la época fría (otoño - invierno) en Córdoba (Battán Horenstein et al. 2010) y Buenos Aires (Centeno et al. 2002), mientras que en la provincia de San Juan (Aballay et al.
2008) sólo se registra durante el verano, estando ausente en Salta (Ayón et al. 2004). Las diferencias regionales descriptas son destacables dado que, en las zonas templado-cálidas C. vicina es un indicador de clima frío mientras que, en la zona NO de Patagonia, de acuerdo a los resultados del presente trabajo y en estudios previos (Armani et al. 2015), la estacionalidad sólo estaría indicada por su dominancia y no por su presencia.

Compsomyiops fulvicrura Robineau-Desvoidy está presente todo el año en Chubut, pero domina en la época cálida (primavera - verano), siendo más abundante durante el verano para el ambiente ribereño, por lo tanto sería también una especie indicadora de estacionalidad.

En cuanto a Lucilia sericata Meigen, es más abundante en otoño, y está presente en Chubut en las cuatro estaciones, coincidiendo con los datos obtenidos para Salta (Ayón et al. 2004) y Córdoba (Battán Horenstein et al. 2010).

Las especies de Sarcophagidae y Muscidae fueron más numerosas durante el verano, y su menor abundancia se registró en invierno, aunque se observa una gran diversidad de especies pero poca abundancia de ejemplares.

Durante el verano se registró a Stevenia deceptoria (Loew, 1847) asociada a los cadáveres de cerdo en descomposición. Esta especie junto a Melanophora roralis (Linnaeus, 1758) son las únicas de la familia Rhinophoridae presentes en la región Neotropical, y fueron registradas en Argentina para la provincia de Buenos Aires (Blanchard y De Santis 1975; Mulieri et al. 2010). Stevenia deceptoria es una especie que 


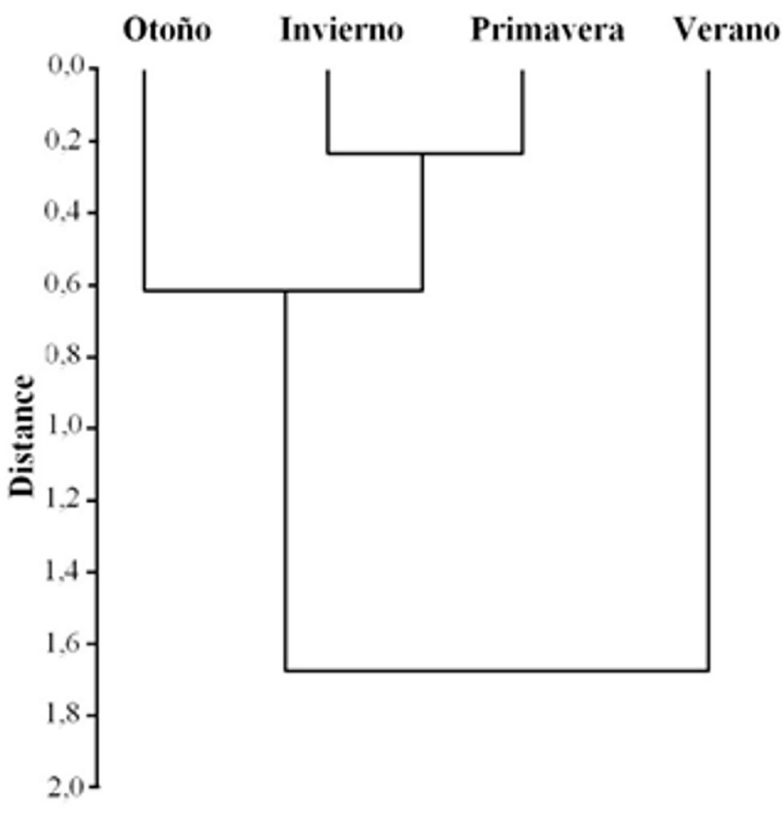

Figura 2. Dendrograma mostrando la relación de similitud (Jaccard) entre las estaciones del año y los índices de diversidad de Shannon y de riqueza de Margalef

parasita isópodos terrestres, por lo tanto su ocurrencia depende de la presencia de sus hospedadores (Mulieri et al. 2010). Esto explicaría el registro de ejemplares adultos hallados en asociación a los modelos porcinos experimentales para el ambiente ribereño.

Dentro de Coleoptera, los Dermestidae fueron más abundantes en verano e invierno a diferencia de lo observado en Salta (Ayón et al. 2004) y en Buenos Aires (Centeno et al. 2002).

En tanto que los Staphylinidae fueron más abundantes y diversos en invierno, a diferencia de los resultados obtenidos en Córdoba (Battán Horenstein et al. 2010), Salta (Ayón et al. 2004), Buenos Aires (Centeno et al. 2002) y San Juan (Aballay et al. 2008).

Scotobius pilularius (Germar, 1823) fue la especie de Tenebrionidae más abundante y presente todo el año, aunque domina en otoño en el ambiente ribereño de Chubut. En el invierno, los Antichidae son muy abundantes, registrándose solamente en la época fría (otoño - invierno).

Entre los Hymenoptera, Solenopsis sp. (Formicidae) fue la especie dominante durante todo el año. Los ejemplares fueron observados depredando larvas y adultos de Calliphoridae (Diptera) sobre los cadáveres de cerdo en todas las estaciones del año.

\section{Conclusiones}

El presente trabajo aporta información de base sobre las características y duración de las distintas etapas del proceso de descomposición en un ambiente ribereño, lo que permitirá conocer en profundidad dicho proceso aportando información notable en casos forenses humanos en la zona de estudio. Se comprobó que la estacionalidad de Calliphora vicina en la provincia de Chubut, está marcada por su dominancia y no por su presencia. Esta característica posiblemente se deba a que la zona de estudio presenta un
Tabla 2. Valores de los índices de diversidad (Shannon) y riqueza específica (Margalef) durante las cuatro estaciones del año. Referencias Pri: primavera, Ver: verano, Inv: invierno, Oto: otoño.

\begin{tabular}{ccccc}
\hline Índice & Pri & Ver & Oto & Inv \\
\hline Shannon & 2,182 & 2,349 & 1,682 & 2,398 \\
Margalef & 4,744 & 6,38 & 4,827 & 4,667 \\
\hline
\end{tabular}

clima templado-frío, a diferencia de lo que ocurre con otras localidades argentinas con temperaturas templado-cálidas donde C. vicina está presente solo en la época fría (otoñoinvierno). Se determinó que la Compsomyiops fulvicrura es indicadora de estacionalidad en la época cálida (primavera - verano) en la zona NO de la provincia de Chubut, Argentina. Se aporta información relevante en relación a las especies de insectos sarcosaprófagos que pueden ser utilizados en la zona NO de la provincia de Chubut, Argentina y más específicamente en ambientes ribereños o fluviales para la estimación de la data de muerte en casos forenses humanos. Se contribuyó a establecer una línea de base sobre el estudio de la fauna cadavérica en la región, permitiendo ampliar el conocimiento de la biodiversidad de artropodofauna en el ambiente ribereño del río Chubut, y más específicamente en el Valle Inferior del mencionado río.

\section{Agradecimientos}

Agradecemos a Ramiro Seguel por la colaboración en las tareas de campo y laboratorio. A Dr. Pablo Mulieri (MACN), Dr. Luciano Patitucci (MACN), Dr. Germán Cheli (CENPAT), Dr. Gustavo Flores (IADIZA), Dr. Fernando Aballay (IADIZA), Dr. Norma Gorosito (UNQ) y a Dr. Chani-Posse (IADIZA) CONICET por la identificación de ejemplares. Agradecemos a CONICET y la Secretaria de Ciencia, Tecnología e Innovación Productiva de la provincia del Chubut por financiar la beca doctoral que permitió esta investigación; al CENPAT por proveer la infraestructura para realizar los muestreos experimentales y trabajo de laboratorio. A ALUAR por el financiamiento para realizar parte de los muestreos experimentales.

\section{Literatura citada}

ABALlAY, F. H.; MURÚA, A. F.; ACOSTA, J. C.; CENTENO, N. 2008. Primer registro de artropodofauna cadavérica en sustratos humanos y animales en San Juan, Argentina. Revista de la Sociedad Entomológica Argentina 67 (3-4): 157-163.

ABALLAY, F., FERNANDEZ CAMPÓN, F.; MULIERI, P.; URQUIZA, S. 2011. Sarcophagidae (Diptera) de importancia forense en la puna de Catamarca, Argentina: la ovoviviparidad como ventaja en condiciones de extrema aridez. Revista de la Sociedad Entomológica Argentina 70 (3-4): 255-266.

ABALLAY, F. H.; DOMINGUEZ; M. C.; FERNANDEZ CAMPÓN, F. 2012. Adult Fanniidae associated to pig carcasses during the winter season in a semiarid environment: Initial examination of their potential as complementary PMI indicators. Forensic Science International 219: 284-284.

ANDERSON, G. S.; VANLAERHOVEN, S. L. 1996. Initial studies on insect succession on carrion in southwestern British Columbia. Journal of Forensic Science 41: 617-625.

ARMANI, A. P.; CENTENO, N. D.; DAHINTEN, S. 2015. Primer estudio de artropodofauna cadavérica sobre modelos experimentales porcinos en el noreste de la provincia de Chubut, Ar- 
gentina. Revista de la Sociedad de Entomología Argentina 74 (3-4): 123-132.

AYÓN, R.; CORRONCA, J. A.; CENTENO, N. D. 2004. Sucesión de artrópodos y diversidad de dípteros en cadáveres de cerdos en área rural de campo Quijano Salta (Argentina). En: II Reunión Binacional Ecología, Mendoza pp. 195.

AYÓN, R. 2013. Sucesión de artrópodos carroñeros en cadáveres de cerdo (Sus scrofa) en la provincia de Salta, Argentina: análisis de los posibles factores que determinan su cambio. Tesis Doctoral, Universidad Nacional de Quilmes. 170 p.

BATTÁN HORENSTEIN, M.; LINHARES, A. X. 2011. Seasonal composition and temporal succession of necrophagous and predator beetles on pig carrion in central Argentina. Medical and Veterinary Entomology 25: 395-401.

BATTÁN HORENSTEIN, M.; SALVO; A. 2012. Community dynamics of carrion flies and their parasitoids in experimental carcasses in central Argentina. Journal of Insect Science 12: 8.

BATTÁN HORENSTEIN, M.; ARNALDOS, M. I.; ROSSO, B.; GARCIA, M. D. 2005. Estudio preliminar de la comunidad sarcosaprófaga en Córdoba (Argentina): aplicación a la entomología forense. Anales de Biología 27: 191-201.

BATTÁN HORENSTEIN, M.; LINHARES, A. X.; ROSSO, B.; GARCÍA, M. D. 2007. Species composition and seasonal succession of saprophagous calliphorids in a rural area of Córdoba, Argentina. Biology Research 40: 163-171.

BATTẢN HORENSTEIN, M.; LINHARES, A. X.; ROSSO, B.; GARCÍA, M. 2010. Decomposition and dipteran succession on pig carrion in central Argentina: ecological aspects and their importance to forensic science. Medical and Veterinary Entomology 24: 16-25.

BATTÁN HORENSTEIN, M.; ROSSO, B.; GARCÍA, M. D. 2012. Seasonal structure and dynamics of sarcosaprophagous fauna on pig carrion in a rural area of Cordoba (Argentina): Their importance in forensic science. Forensic Science International 217 (1-3): 146-156.

BENECKE, M. 2001. A brief history of forensic entomology. Forensic Science International 120: 2-14.

BLANCHARD, E. ; DE SANTIS, L. 1975. Primera lista anotada de Oestromuscarios entomófagos argentinos. Revista de Investigaciones Agropecuarias, INTA. Serie V Patología Vegetal Vol. XII N ${ }^{\circ} 1: 7.76$.

BYRD, J. H.; CASTNER, J. L. 2001. The utility of arthropods in legal investigations. CRC Press, Boca Ratón.

CASTILLO MIRALBÉS, M. 2002. Estudio de entomofauna asociada a cadáveres en Aragón. Sociedad Entomológica Aragonesa vol. $6,87 \mathrm{p}$.

CENTENO, N. 1998. Extensión del rango de distribucional de Chrysomya spp. en la Argentina (Diptera: Calliphoridae). Revista de la Sociedad Entomológica Argentina 57 (1-4): 137-138.

CENTENO, N.; MALDONADO, M.; OLIVA, A. 2002. Seasonal patterns of arthropods occurring on sheltered and unsheltered pig carcasses in Buenos Aires Province (Argentina). Forensic Science International 126: 63-40.

DADOUR, I.; COOK, D.; FISSIOLI, J.; BAILEY, W. 2001. Forensic entomology: application, education and research in Western Australia. Forensic Science International 120 (1-2): 48-52.

MARILUIS, J.; MULIERI, P. 2003. The distribution of the Calliphoridae in Argentina (Diptera). Revista de la Sociedad Entomológica Argentina 62 (1-2): 85-97.
MARCHENKO, M. I. 2001. Medicolegal relevance of cadaver entomofauna for the determination of the time of death. Forensic Science International 120 (1-2): 89-109.

MARIANI, R.; GARCÍA-MANCUSO, R.; VARELA, G. L.; INDA, A. M. 2014. Entomofauna of a buried body: Study of the exhumation of a human cadaver in Buenos Aires, Argentina. Forensic Science International 237: 19-26.

MARTÍNEZ, E.; DUQUE, P.; WOLFF, M. 2007. Succession pattern of carrion-feeding insects in Paramo, Colombia. Forensic Science International 166: 182-189.

MULIERI, P.; MARILUIS, J. C.; PATITUCCI, L. 2010. Review of the Sarcophaginae (Diptera: Sarcophagidae) of Buenos Aires Province (Argentina), with a key and description of a new species. Zootaxa 2575: 1-37.

OLIVA, A. 1997. Insectos de interés forense de Buenos Aires (Argentina). Primera lista ilustrada y datos bionómicos. Revista del Museo Argentino de Ciencias Naturales "Bernardino Rivadavia", Entomología 7 (2): 13-59.

OWEN, J.; HUGHES, G.; HERRERO, G.; SERDÁ, A.; GRIZNIK, M. 2005. IGEOPAT, Párrafos Geográficos $N^{*} 4$ "Manejo Integral del Estuario del Rio Chubut".

PAYNE, J. A. 1965. A summer carrion study of the baby pig Sus scrofa Linnaeus. Ecology 46 (5): 592-602.

SALAZAR ORTEGA, J. 2008. Estudio de la entomofauna sucesional asociada a la descomposición de un cadáver de cerdo doméstico (Sus scrofa) en condiciones de campo. Universitas Sciantarium Revista de la Facultad de Ciencias 13 (1): 21-32.

SEGURA, N. A.; USAQUÉN, W.; SÁNCHEZ, M. C.; CHUAIRE, L.; BELLO, F. 2009. Succession pattern of cadaverous entomofauna in a semi-rural area of Bogotá, Colombia. Forensic Science International 187: 66-72.

SEGURA, N. A.; BONILLA, M. A.; USAQUEN, W.; BELLO, L. 2011. Entomofauna resource distribution associated with pig cadavers in Bogota DC. Medical and Veterinary Entomology 25: 46-52.

TABOR, K.; FELL, R.; BREWSTER, C. 2005. Insect fauna visiting carrion in Southwest Virginia. Forensic Science International 150 (1): 73-80.

WOLFF, M.; URIBE, A.; ORTIZ, P.; DUQUE, A. 2001. A preliminary study of forensic entomology in Medellin, Colombia. Forensic Science International 120: 53-59.

ZANETTI, N. I.; VISCIARELLI, E. C.; CENTENO, N. D. 2015. Associational patterns of scavengers beetles to decomposition stages. Journal Forensic Science 60: 919-927.

Recibido: 22-mar-2016 • Aceptado: 16-may-2017

Citación sugerida:

ARMANI, A. P.; DAHINTEN, S.; CENTENO, N. 2017. Artropodofauna cadavérica asociada a cerdo doméstico (Sus scrofa) en un ambiente ribereño en Chubut, Argentina. Revista Colombiana de Entomología 43 (2): 262-267. Julio - Diciembre 2017. ISSN 0120-0488. 